\title{
Electric, Magnetic and Gravitation Field of the Earth
}

\author{
Alexander Ivanchin \\ Orpheus Ltd., Tomsk, Russia \\ Email: al.g.ivanchin@gmail.com
}

How to cite this paper: Ivanchin, A. (2017) Electric, Magnetic and Gravitation Field of the Earth. Journal of Geoscience and Environment Protection, 5, 66-79. https://doi.org/10.4236/gep.2017.512005

Received: October 30, 2017

Accepted: December 15, 2017

Published: December 18, 2017

Copyright $\odot 2017$ by author and Scientific Research Publishing Inc. This work is licensed under the Creative Commons Attribution International License (CC BY 4.0).

http://creativecommons.org/licenses/by/4.0/

\section{(c) (i) Open Access}

\begin{abstract}
In the article a theory of the origin of the magnetic field of the Earth (MFE) caused by the phenomenon of ferromagnetism in the mantle has been proposed. Due to the pressure the mantle substance changes from the dielectric state to the metal one. As a result, there appear free electrons, whose magnetic moment leads to the formation of the magnetic field of the Earth. The above phase transition manifests itself by the Moho. It is proposed that not only the measurement of the dipole component of the magnetic field be made, as it is done nowadays, but also that of the quadrupole and octapole components. These measurements will provide information on the origin of the MFE and its distribution through the Earth volume. It has been shown that the electric field of the Earth (EFE) cannot be produced by the movement of the mobile charges in principle. The EFE is produced by bound electrons. A hypothesis has been proposed that the electric constant has different values for the proton-proton, electron-electron and proton-electron interactions. The relative value of this difference is quite small of the order of $10^{-28}$ and, for this reason, it has not been found experimentally. The difference in the interaction results in gravitation, the attraction of the electroneutral masses. Gravitation is a variety of electrostatics. As a result of this difference, the electrons are gravitationally stronger attracted to the electroneutral mass than the protons. The gravitating mass attracts surplus electrons, which lead to the formation of the EFE. In the atmosphere around the negatively charged Earth there are positive charges screening the Earth charge. This state is equilibrium; the positive charge of the atmosphere cannot penetrate into the condensed substance and neutralize the surplus negative charge of the Earth, which would increase the system energy.
\end{abstract}

\section{Keywords}

Magnetic Field of the Earth, Electric Field of the Earth, Gravitational Field of the Earth 


\section{Introduction}

At present there are no well-founded theories of the origin of the Magnetic Field of the Earth (MFE) and the Electric Field of the Earth (EFE). The proposed hypotheses contradict the laws of physics and are, in essence, dynamic, as they relate the existence of the fields to some processes occurring in time. According to them, all processes slow down with time and reach equilibrium, i.e. a stable state. The MFE is explained by an electric current in the nucleus of the Earth. However, it exists during geological periods of time, which rules out this variant of origin.

The same holds true for the EFE. It is not possible to evaluate the time of existence of the EFE using the methods of paleomagnetism for the MFE, since the carriers of paleo-electrostatics have not been found yet. However, the EFE is sure to have existed for hundreds of years. Its relaxation time amounts to minutes. There arises a problem of supplying the Earth's charge. It is absolutely groundless to attribute it to thunderstorms. The EFE is a global stationary phenomenon, whereas thunderstorms are local. If there forms an electric charge in a cloud, then due to the induction near the surface of the Earth directly under the cloud there appears an electric charge of the opposite sign equal to that of the cloud. Separation of charges during a thunderstorm is a local phenomenon, whereas the charge in the Earth is concentrated in the layer near the surface. A lightning is responsible for their discharge; therefore, it is not correct to state that thunderstorms produce the charge. Thunderstorms are not possible without lightnings, and lightnings eliminate the separation of charges. Part of lightnings take place between clouds, i.e. there is separation of charges between clouds rather than between a cloud and the Earth. Therefore, it is not right to attribute the production of the Earth's charge to thunderstorms. Here static models of the formation of the MFE and the EFE are proposed.

\section{The Magnetic Field of the Earth}

The magnetic moment of the Earth is $7.86861 \times 10^{27} \mathrm{~A} \cdot \mathrm{m}^{2}$ [1]. The origin of the MFE is not clear, the main hypothesis being an eddy current in the Earth's nucleus. However, without generation the current would cease in fractions of a second, whereas the MFE has existed during the geological periods of time. There are no direct or indirect proofs of the above hypothesis. It contradicts to the laws of physics because one has to answer the following questions: What generates the current and where does the energy come from? Where does the heat produced by the current go? Or is the Earth's nucleus in the superconducting state? It is absolutely true that the temperature in the nucleus is thousands of degrees of Kelvin. At such temperatures superconductivity is not observed. It should also be noted that superconductivity occurs in solids with a crystalline structure, whereas the nucleus is liquid. There are also some other conflicts of the above hypothesis with physics.

Another hypothesis has been put forward concerning the origin of the mag- 
netic field due to there being an iron nucleus in the ferromagnetic state. However, liquid iron does not possess the properties of ferro-magnetism.

In the theoretical aspect, the problem of the MFE has not been well studied yet, which looks strange in the background of the high level of the development of the magnetic theory in physics. For centuries the parameters of the MFE intensity have been measured and charts of their values have been published [2]. The Legendre spherical functions are used for the mathematical processing of measurement results. As is known, these functions form a complete system of orthogonal polynomials necessary for the expansion of an arbitrary function defined at the surface of a sphere [3]. At first glance, it is all right, the expansion coefficients into a series applying the Legendre polynomials are found, i.e. the expansion in terms of multi-poles. However, there is the reverse of the medal.

Measurements of the MFE are made only with the help of dipole-a magnetic pointer. A dipole responds to the dipole component of the field. Its energy of interaction with multi-poles of any order other than a dipole is zero, therefore, the information acquired concerns only the dipole component of the MFE. That means that the coefficients in the expansion of the MFE applying the Legendre polynomials are purely abstract values having no physical sense, and they cannot be compared with existing multi-poles, whose values have not been measured. They cannot give information on the real structure of the MFE. To measure the quadrupole component of the MFE, it is necessary to use the magnetic quadrupole as a sensor, however, I am not aware of such measurements. The same is true for the multi-poles of a higher order. It is necessary to measure them to understand the real structure of the MFE, since the dependence of the interaction energy of multi-poles differs from that of dipoles. Therefore, by measuring it one can get information on the depth of the magnets' position, their orientation and distribution through the Earth volume, etc. At the moment there is no such information.

Besides, the above expansion is applicable only for the field of a point source of magnetism located in the center of the Earth. For a volume source it would be necessary to integrate with respect to the volume of the region occupied by the magnet. To do this, one needs the information on the distribution of the magnets through the volume, which is missing.

The dipole component of a point magnetic source has the axis of symmetry, i.e. the magnetic poles must be antipodes similarly to the geographical ones. However, this is not the case. The coordinates of the magnetic poles are in the northern hemisphere in latitude $85^{\circ} 54^{\prime}$ North and Long $147^{\circ} 00^{\prime} \mathrm{W}$; in the southern hemisphere in latitude $64^{\circ} 24^{\prime}$ South and Long $137^{\circ} 06^{\prime} \mathrm{E}$. The antipode of the magnetic pole in northern hemisphere is the point with the coordinates in latitude $85^{\circ} 54^{\prime}$ South and Long $43^{\circ} \mathrm{E}$. The angle between the verticals at the magnetic poles is $153^{\circ}$ rather than $180^{\circ}$. If the MFE source is not a point one, then the field is produced by the magnets distributed through the Earth's volume and the location of the magnetic poles can be arbitrary. For instance, U-shaped magnets have the poles revolved relative to each other through an angle of $180^{\circ}$. 
The isocline and isogonic lines measured do not possess the axis of symmetry. Therefore, it is necessary to consider the volume distribution of the magnetic sources.

Ferromagnetic Hypothesis of the Magnetic Field of the Earth. It is known from physics that under certain thermodynamic conditions some substances can change to the ferromagnetic state, their magnetic field produced due to the electron magnetic moments. There exists the Curie temperature below which this magnetization is observed. As noted earlier [4] [5] a probable reason for the existence of the Moho in the lithosphere is the phase transition related to the lithosphere pressure. Due to this phase transition there may appear conditions for ferro-magnetism, with the mantle substance changing to the magnetic state. The next known phase transition is the nucleus boundary, where the hard mantle changes to a liquid and, probably, loses the property of ferro-magnetism. Thus, the MFE may be produced by the mantle. It is possible that not the whole mantle but only its part up to a certain depth, where under the influence of a temperature rise, there occurs the so-called phase transition of a second type, when the substance loses the property of ferro-magnetism and the elastic moduli do not change. For this reason, the phase transition of the second type is not registered by the elastic waves. In the geological aspect, the ferro-magnetism of rocks is a well-known fact.

The Moho is a phase transition during which the substance from the dielectric state under pressure changes to the metal one, when there appear, the so-called, sharing (or, otherwise, free) electrons. At this transition the electric conductivity increases by several orders of magnitude and so does the coefficient of thermal conductivity [6]. The pressure at the Moho at which the above phase transition occurs is of the order of $10^{9} \mathrm{~Pa}$. Experimental study of the thermodynamic properties of the rocks at the above pressure is of great interest for geophysics. However, at the modern level of experiment the above pressure values are unattainable. Nowadays it is not clear what values should be measured to experimentally prove the ferromagnetic state of the mantle. Even if it were possible to bore to the Moho, the phase transition might not be found, since around the borehole the pressure would be released and the rock from the ferromagnetic state would change to the usual one. It may be possible to answer this question if the multi-pole components of the MFE are measured.

The radius of the Moon is $1737 \mathrm{~km}$, almost 4 times less than that of the Earth, and the free fall acceleration on the Moon surface is 6 times less than on the Earth. As a result, the phase transition of the Moon will be, at best, 6 times deeper than that of the Earth. On the Earth under the continents the Moho is at a depth of the order of $70 \mathrm{~km}$, then on the Moon it will be at a depth no less than $500 \mathrm{~km}$, and, as a matter of fact, even deeper. The radius of the Earth is $6371 \mathrm{~km}$, so the value of $70 \mathrm{~km}$ is small as compared to the radius of the Earth. Therefore, the free fall acceleration at a depth of $70 \mathrm{~km}$ is, in fact, the same as on the surface of the Earth. For the Moon it will be different, since $500 \mathrm{~km}$ is almost a third of the Moon's radius, and therefore, at this depth the value of the free fall accelera- 
tion will be much less than on the Moon's surface. This factor considerably decreases the pressure and the Moho for the Moon, if it exists at all, will be located much deeper. Besides, as shown in [4] [5] the pressure decreases also due to the redistribution of the stresses between normal and shift stresses. Thus, if the Moho on the Moon does form, it is at a larger depth, not far from the Moon's center, which explains the fact that the Moon has no magnetic field. The phase transition into the ferromagnetic state either does not take place on the Moon or occurs at a large depth. As a result, the volume of the ferromagnetic mass is at a large distance from the Moon surface, and the strength of the magnetic field decreases in proportion to the cube of distance, which results in even a larger decrease in the magnetic field on the surface of the Moon. Thus, if the magnetic field does exist on the surface of the Moon, it is by several orders of magnitude less than on the surface of the Earth.

\section{Electrostatics and Gravitation}

The Electric Field of the Earth. The strength of EFE near the surface of the Earth is about $130 \mathrm{~V} / \mathrm{m}$, at a height of tens of kilometers it drops down to millivolt per meter. The Earth carries a negative charge of the order of $1.5 \times 10^{5} \mathrm{C}$ and the atmosphere-the same but the positive one. For some reason, these charges cannot neutralize each other in spite of the fact that it takes several minutes [7].

Several variants of charging have been proposed, or to be more correct, one variant with variations. Charging occurs due to the movement of charged particles, e.g. drops of water. In some hypotheses these drops rise with the convective streams upward, in others, vice versa, they fall down together with rain. During the above charging there happens separation of charges while the total charge remains unchanged. Due to the electric induction under the charged cloud near the surface of the Earth there is a charge of the opposite sign but of the same value. These charges can discharge through a lightning or without it due to the electric conductivity of the air. As a result, no global charging occurs. The EFE theories are critically examined in [7].

Let two elementary charges ${ }^{1} q_{1}$ and $q_{2}$ be located at points $x=-a$ and $x=+a$ on the axis of abscissa, they may differ in the sign and be the same in the modulus, i.e. $\left|q_{1}\right|=\left|q_{2}\right|=e$, here $e$-is a proton charge. These charges, according to the classical theory, produce the strength at a point with the coordinates $\boldsymbol{r}=\{x, y, z\}$

$$
\boldsymbol{E}=\frac{1}{4 \pi \varepsilon}\left(\frac{q_{1}}{r_{+}^{3}} \boldsymbol{r}_{+}+\frac{q_{2}}{r_{-}^{3}} \boldsymbol{r}_{-}\right)
$$

Here it is designated

$$
\begin{aligned}
& \boldsymbol{r}_{-}=\{x+a, y, z\}, r_{-}=\sqrt{(x+a)^{2}+y^{2}+z^{2}} \\
& \boldsymbol{r}_{+}=\{x-a, y, z\}, r_{+}=\sqrt{(x-a)^{2}+y^{2}+z^{2}}
\end{aligned}
$$

${ }^{1}$ Elementary charge is the charge of a proton. 
The values of $r_{-}$and $r_{+}$are the distances from the charges to point $r$. The electrostatic energy density, according to the classical theory, produced by the charges will be written as

$$
w=\frac{\varepsilon}{2} \boldsymbol{E} \cdot \boldsymbol{E}=\frac{1}{32 \pi^{2} \varepsilon}\left(\frac{q_{1}}{r_{+}^{3}} \boldsymbol{r}_{+}+\frac{q_{2}}{r_{-}^{3}} \boldsymbol{r}_{-}\right) \cdot\left(\frac{q_{1}}{r_{+}^{3}} \boldsymbol{r}_{+}+\frac{q_{2}}{r_{-}^{3}} \boldsymbol{r}_{-}\right)=w_{1}+w_{2}+w
$$

Here $w_{1}$ and $w_{2}$ denote the density of the intrinsic energy of the charges

$$
w_{1}=\frac{e^{2}}{32 \pi^{2} \varepsilon^{2} r_{+}^{4}}, \quad w_{2}=\frac{e^{2}}{32 \pi^{2} \varepsilon^{2} r_{-}^{4}}
$$

and $w$ is the energy of the charge interaction

$$
w_{i}=\frac{e^{2}}{16 \pi^{2} \varepsilon^{2}} \frac{\boldsymbol{r}_{-} \cdot \boldsymbol{r}_{+}}{r_{-}^{3} r_{+}^{3}}
$$

The sign of $w_{i}$ is determined by the signs of the interacting charges. If the signs of the charges are the same, then in (5) the sign is plus, if they are different, it is minus. The densities of the intrinsic energies (4) do not depend on the distance between the charges, they become zero under differentiation with respect to this distance. Therefore, they are not considered further. Integrating (5) with respect to the whole volume $V$, one obtains the total interaction energy produced by the charges

$$
\begin{aligned}
W & = \pm \varepsilon \iiint_{V} w_{i} \mathrm{~d} V= \pm \frac{e^{2}}{16 \pi^{2} \varepsilon} \iiint_{V} \frac{\boldsymbol{r}_{-} \cdot \boldsymbol{r}_{+}}{r_{-}^{3} r_{+}^{3}} \mathrm{~d} V \\
& = \pm \frac{e^{2}}{16 \pi^{2} \varepsilon} \iiint_{V} \frac{x^{2}-a^{2}+y^{2}+z^{2}}{\left[(x-a)^{2}+y^{2}+z^{2}\right]\left[(x+a)^{2}+y^{2}+z^{2}\right]} \mathrm{d} V \\
& = \pm \frac{e^{2}}{8 \pi \varepsilon a}
\end{aligned}
$$

Calculation of the integral is shown in [8] [9]. The denominator of the Formula (6) contains the multiplier $8 \pi$, whereas the standard formula has $4 \pi$. This difference is related to the fact that here half the distance $a$ between the charges is used.

Let us assume that the electric interaction is somewhat different for different types of charges: for positive ones it will be written in the standard form (5) with the sign of plus

$$
\dot{w}=\frac{e^{2}}{8 \pi \varepsilon a}
$$

and for negative ones

$$
\bar{w}=+(1-\xi) \frac{e^{2}}{8 \pi \varepsilon a}=(1-\xi) \dot{w}
$$

whereas for the opposite ones

$$
\ddot{w}=-(1-\eta) \frac{e^{2}}{8 \pi \varepsilon a}=-(1-\eta) \dot{w}
$$


At $\xi=\eta=0$ Formulas (7), (8), (9) change to the standard formulas of electrostatics. This difference is caused by the fact that the electric constant $\varepsilon$ is somewhat different for the interaction of different types of charges. It is equal to $\varepsilon$ for the interaction of positive charges, $\varepsilon /(1-\xi)$ for that of negative charges and $\varepsilon /(1-\eta)$ for the interaction of the negative charge with the positive one. Integrating (7), (8), (9) over the whole space one obtains the interaction energy in the form

$$
\begin{gathered}
\dot{W}=W \\
\bar{W}=(1-\xi) W \\
\ddot{W}=-(1-\eta) W
\end{gathered}
$$

Here $W$ is given by the relation (6).

Let us assume one atom of hydrogen located at the points $-a$ and $+a$ on the axis of abscissa, i.e. a proton with an electron. The distance $a$ is much larger than the characteristic size of an atom. In this case, it is possible to consider the electrons and the protons of different atoms to be located at the same distance of $2 a$. The interaction energy of these hydrogen atoms consists of the sum of the interaction energies of the proton-proton (10), the electron-electron (11) and the doubled sum of the electron-proton (12). The doubling is caused by the fact that there are two pairs of the electron-proton, whereas there is only one with the same sign. The energy of the intra-atomic interaction of the proton-electron is not taken into account, since it does not depend on the distance between the atoms. Adding (10), (11) and the doubled (12) energies one derives the interaction energy of two hydrogen atoms

$$
\frac{e^{2}(2 \eta-\xi)}{8 \pi \varepsilon a}
$$

The values of $\eta \ll 1$ and $\xi \ll 1$. If it was different, it would be observed in the experiment. They cannot be larger than the error of measurement of the electric constant. At present it has been determined accurate to 0.0001; therefore, $\eta$ and $\xi$ in magnitude are less than the above value. From (13) it follows that at

$$
2 \eta-\xi=0
$$

there is no interaction. At

$$
2 \eta-\xi<0
$$

the interaction energy is negative, and the atoms attract one another. It is this variant that is really fulfilled. If

$$
2 \eta-\xi>0
$$

the interaction energy is positive, and the atoms repel one another.

It should be noted that in the Formulas (8)-(9) it is the electric constant $\varepsilon$ that is different rather than the charge itself. If the proton charge were different in absolute value from the electron charge, then two hydrogen atoms would always repel each other. 
The gravitation energy of the interaction of two hydrogen atoms will be written as

$$
W_{g}=-G \frac{m_{H}^{2}}{2 a}
$$

Here $G$ is the gravitation constant, $m_{H}=m_{p}+m_{e}$ is the mass of a hydrogen atom, $m_{p}$ and $m_{e}$ are the masses of a proton and an electron, respectively, the sign of minus is because gravitation means attraction, therefore, the interaction energy is negative. Equating (13) and (16) one derives

$$
\xi-2 \eta=4 \pi \varepsilon \frac{m_{H}^{2} G}{e^{2}}
$$

It is necessary to take into account one more factor, i.e. a proton and an electron interact with a neutral hydrogen atom in a different way, which results in the excess negative charge of the gravitating sample. Further this excess charge will be referred to as a $g$-charge.

Suppose a hydrogen atom is at the point $x=-a$ and an electron at the point $x=a$. In this case, there are two types of interaction: an electron-electron and an electron-proton. Their interaction energy is found by taking the sum of (8) and (9)

$$
\bar{W}+\ddot{W}=\frac{e^{2}(\eta-\xi)}{8 \pi \varepsilon a}
$$

This energy is a negative value, i.e. the electron is gravitationally attracted to the hydrogen atom (see (29)). The interaction energy of the hydrogen atom and the proton is estimated in the same way as the interaction energy of the electron with the hydrogen atom (18). One derives it in the form

$$
\dot{W}+\ddot{W}=\frac{e^{2} \eta}{8 \pi \varepsilon a}
$$

This value is positive one, and the proton is repulsed hydrogen atom. The difference between (19) and (18) (19) one obtains (13), and the difference between (18) and (19) is the value

$$
-\xi \frac{e^{2}}{8 \pi \varepsilon a}
$$

The above value is negative, i.e. the electron is gravitationally stronger attracted to the electrically neutral atom of hydrogen than the proton. As a result, the gravitating body gets a negative charge. Due to the negative charge, around the gravitating body there forms a cloud of positively charged particles (the Debye cloud), which are attracted to the negative charge and neutralize it at some distance from the Earth. The above state of the negatively charged Earth with the positively charged Debye cloud around it is a stable system existing at minimum energy.

The relative value of the $g$-charge falling, on the average, at one nucleon will be as follows 


$$
\varkappa=\frac{Q m_{H}}{e M} \approx 2.62 \times 10^{-28}
$$

The value of $\varkappa$ does not depend on the body mass, it is the same for all bodies, which is due to the fact that the $g$-charge is proportional to the mass of a body, i.e. $Q / M=$ const .

A neutron can be considered a complex of a proton with an electron, therefore, the gravitation interaction of a hydrogen atom with a neutron does not differ in consideration from the interaction of two hydrogen atoms presented above. Really, a neutron consists of an electron and a proton and falls apart into a proton and an electron. The gravitational interaction of a neutron with an electron can be considered the interaction of a neutral hydrogen atom with an electron (18) and a proton (19), while the gravitational interaction of two neutrons like the one of two hydrogen atoms. As a result, the above interaction of two hydrogen atoms holds true for the interaction of the atoms of any substance and further for any set of atoms that all bodies consist of. Thus, gravitation is a variety of electrostatic interaction.

The Charge of the Earth. If an electron charge is increased by a certain value, e.g. $\varkappa$, then the interaction energy (11) and (12) will become

$$
\begin{aligned}
& \bar{W}^{\prime}=(1+\varkappa)^{2}(1-\xi) W \\
& \ddot{W}^{\prime}=-(1+\varkappa)(1-\eta) W
\end{aligned}
$$

The energy in (22) is positive, while that in (23) is negative. Thus, the negative charge of the Earth is, as if, distributed over each nucleon increasing slightly the electron charge. As a result, among the nucleons there appears the force of electrostatic repulsion. The equilibrium is reached when this force compensates the excess force of the electron attraction produced by the energy (20). Summing up (10), (22), (23), differentiating with respect to the distance and equating the result to zero one derives the following equation

$$
\varkappa=\frac{\xi-\eta}{\xi-1}
$$

The Equations (17) and (24) form a system of two equations with respect to $\eta$ and $\xi$ and solving it one gets

$$
\begin{gathered}
\eta=\frac{1}{1+2 \varkappa}\left[\varkappa+8 \pi \varepsilon(1+\varkappa) \frac{G m_{H}^{2}}{e^{2}}\right] \\
\xi=\frac{1}{1+2 \varkappa}\left[2 \varkappa+4 \pi \varepsilon \frac{G m_{H}^{2}}{e^{2}}\right]
\end{gathered}
$$

Hence one obtains

$$
\beta=2 \eta-\xi=8 \pi \varepsilon \frac{G m_{H}^{2}}{e^{2}}
$$

Substituting here the values of the parameters

$$
\begin{aligned}
& m_{H}=1.674 \times 10^{-27} \mathrm{~kg}, M=5.972 \times 10^{24} \mathrm{~kg}, Q=1.5 \times 10^{5} \mathrm{C} \\
& G=6.674 \times 10^{-11} \mathrm{~N} \cdot \mathrm{m} / \mathrm{kg}^{2}, e=1.602 \times 10^{-19} \mathrm{C}
\end{aligned}
$$


one derives the estimates

$$
\eta=2.62388 \times 10^{-28}, \quad \xi=5.24776 \times 10^{-28}, \quad \beta=1.62073 \times 10^{-36}
$$

The value of $\beta$ is very small as compared to $\eta$ and $\xi$, which is caused by a slight difference in the attraction of the proton and the electron to the hydrogen atom given by the Formula (20).

The proposed interaction mechanism disagrees with the modern concepts of electrostatics, according to which an electron does not interact with a neutral hydrogen atom. A different value of the electric constant for the interaction of the opposite charges leads to the interaction of an electron and a proton with a neutral hydrogen atom, which changes with the distance in the same way as the gravitation potential. The above system is in stationary equilibrium, the positive charge from the Debye cloud cannot relax the negative charge in the gravitating mass, since it would increase the potential energy of the system. This is really observed on the Earth - the stationary negative charge of the Earth and the positive charge above its surface.

Hence it follows from this article and [9] that in nature there exists nothing but the electric field.

\section{The Debye Cloud}

The value of

$$
n_{e}=\frac{Q}{e}
$$

is the number of the electrons producing the $g$-charge of the Earth. Then

$$
\frac{M}{n_{e}} \sim 10 \mathrm{~kg}
$$

is the mass falling within one electron of the $g$-charge, i.e. the mass of the substance of $10 \mathrm{~kg}$ keeps one uncompensated electron. The value of

$$
s_{e}=\frac{4 \pi R^{2}}{n_{e}} \sim 8 \times 10^{-10} \mathrm{~m}^{2}
$$

is the area of the Earth's surface falling within one electron of the $g$-charge and it is approximately equal to the area of a square of the side of $0.03 \mathrm{~mm}$. Here $R=6.3 \times 10^{6} \mathrm{~m}$ is the radius of the Earth. These examples are given to show the orders of the magnitudes characteristics the charge of the Earth. Thus, the gravitation of the planets produces their electric negative charge that results in the cloud of the positive charge around the planet. When a charged particle with the charge $q$ travels the distance $h$ in the electric field with the strength $E$ along the strength line, it performs work

$$
e E h
$$

According to the molecular-kinetic theory, due to the collisions with the atoms of the air or the surface of the Earth, the positive ions possess a kinetic energy equal, on the average, to $k T / 2$ per degree of translation, including the 
vertical component. Here $k=1.38065 \times 10^{-23} \mathrm{~J} / \mathrm{K}$ is the Boltzmann constant, $T$ is the absolute temperature. Equating this kinetic energy to the work (31) one derives the height of the ion rise due to the kinetic energy in the electric field of the Earth for the temperature $T=300 \mathrm{~K}$ :

$$
h=\frac{k T}{2 e E} \sim 0.1 \mathrm{~mm}
$$

This estimate gives the thickness of the Debye cloud if there were no atmosphere on the Earth. The presence of the atmosphere leads to the fact that the Debye cloud covers tens of kilometers in height. The ions, when colliding with the air molecules, exchange the energy, and an ion of the mass $m$ rising to a certain height $h$ and losing, due to this, its kinetic energy $m g h$, increases it again in the collision with an air molecule and will be able to rise at a larger height. The length of the ion free path is much less than the height of the atmosphere. Therefore, the ion interaction with the atmospheric atoms as well as the macroscopic motion of the air will affect the ion distribution in height.

The ascent and descent of the air carry along the positive ions resulting in a change of the EFE strength and produce the difference in the potentials along the surface of the Earth leading to the appearance of telluric currents. The atmosphere contains the ions of both signs formed as a result of the ionizing caused by the ultraviolet and cosmic rays as well as by the natural radioactivity, etc. These ions are affected by the Lorentz force when they move with an air current, for instance, with wind or under convection in the magnetic field of the Earth, there appears an electromotive force and an electric current. The direction of the current is determined by the mutual orientation of the vector of the magnetic field strength and that of the speed of the air, and it can have both the vertical and the horizontal components. On the one hand, this current results in the potential difference along the surface of the Earth and is one more source of telluric currents. On the other hand, it can lead to the separation of the charges in the atmosphere both along the vertical and the horizontal as well as to the formation of lightnings. Charges of large values are not formed at a small height above the surface of the Earth due to the Earth proximity. Its high electrical conductivity produces a shunting effect suppressing the separation of charges. Regions charged up to high values of the potential can form in the atmosphere only at a rather large height.

The mass of the Moon is 81 times less than that of the Earth, its radius is $r_{L}=1700 \mathrm{~km}$, so the electric charge of the Moon must be approximately $Q_{L} \sim 1000 \mathrm{C}$. Then the electric field strength on the surface of the Moon must be as follows

$$
E_{L}=\frac{Q_{L}}{4 \pi \varepsilon r_{L}^{2}} \sim 3 \mathrm{~V} / \mathrm{m}
$$

and the height of the ascent according to (32) for $T=400 \mathrm{~K}$ will be $\sim 6 \mathrm{~mm}$. Since on the Moon there is no atmosphere, this value estimates the thickness of the Debye cloud for the Moon on the light side. On the dark side, where the sur- 
face temperature is much lower, the thickness of the Debye cloud will be several times less. At a height of several centimeters and more from the surface of the Moon the strength of its electric field will be, practically, zero.

The character of the distribution of the charge of the Earth through the volume is of great importance to prove the correctness of the hypothesis of the EFE proposed here. The hypotheses of charging imply that the electrons are mobile, i.e. they are conduction electrons. According to the law of electricity, a charge produced by the above electrons in a conductor must be concentrated on the surface of a sphere. Such distribution of the EFE charges would result in the charge concentration on projecting objects, e.g. mountain tops, metallic high-rise building constructions, etc. Near them the EFE strength would increase several times as compared to lowland. In contrast with this, in deep cavities and canyons the EFE strength would sharply decrease down to zero. There would appear other effects related to the surface charge. However, nothing of the kind has been noted, so it is incorrect to consider that the EFE is produced by the mobile electrons. There are no contradictions of the kind in the hypothesis proposed here. If the charge of the Earth was produced by the mobile electrons, then there would be no EFE under the surface of the water. It is enough to measure the EFE under the surface of the water to determine the type of the electrons producing the EFE. If the electric strength at a depth of several meters does exist, it means that the charge of the Earth is produced by immobile electrons. If there is no electric strength, it is produced by conduction electrons. It is also possible to make similar measurements at a depth below the free surface of the Earth on the dry land.

There are phenomena caused by the conduction electrons and observed under a thundercloud, for instance, St. Elmo's fire and the others. If there are no conditions for separation of the mobile charges, i.e. electric charging, no St. Elmo's fire is observed either. However, the electric field of the Earth produced by the bound $g$-electrons does exist.

\section{Discussion}

The study presented here shows that in order to understand the origin of the EFE and the MFE, it is necessary to reject the dynamic theories of their origin that contradict the known and reliably established physical laws and turn to the static theories, in which the existence of these fields is accounted for by the fact that for physical reasons, the minimum complete system energy is reached with these fields. The same principle of the minimum system energy was earlier used to establish the equilibrium field of the mechanical stresses in the mantle [4] [5].

To study the structure of the EFE and the MFE, it is necessary to perform measurements using sensors responding to multipole components. At present only the monopole component is measured for the EFE, with no question arising on the existence of the dipole, quadrupole and other components of the EFE. As for the MFE, the open question is if there exists the monopole magnetic compo- 
nent of the Earth. A magnetic pointer is a dipole, so it does not respond to the monopole component. The problem of designing a sensor responding to the monopole component has not been solved yet. Measurement of these values would give more detailed information on the structure of the EFE, the MFE and the Earth as well as on the processes occurring in it.

The difference of the electric constant for different charges is caused by the vacuum properties rather than by the charge properties. It is impossible to derive the gravitational interaction if one assumes that the positive and the negative elementary charges are not equal in absolute value. If it was the case, two hydrogen atoms would always repel due to the uncompensated elementary charge.

The electric and magnetic fields are produced not only by the elementary charges, but also appear during the movement of the electric charges. The electric constant which accompanies these fields is of great interest, since it is related to the electric constant for the elementary charges. The solution of this problem can lead to antigravitation.

\section{Conclusion}

From the article presented it follows that there is close interdependence between the electric and gravitational fields. According to [9], the magnetic field is the manifestation of the properties of the electric field. Thus, all the three fields, the electric, magnetic and the gravitational ones, are different manifestations of the electric field. The gravitational field is caused by the properties of the vacuum that responds differently to the positive and negative charges. It should be noted that this difference is weak and becomes pronounced with large masses of matter resulting in separation of charges, which does produce the electric field of the Earth. Other celestial bodies must also possess these fields. Therefore, the interconnection between the electric and gravitational fields should be taken into account when the processes occurring on the sun, stars and planets are considered.

\section{References}

[1] Kikoin, I.K., Ed. (1974) Tanlicy fizicheskih velichin. [Tables of Physical Values.] Atomizdat, Moscow. (In Russian)

[2] NOAA National Centers for Environmental Information (2016) Website of the US Department of Commerce. https://www.ngdc.noaa.gov/geomag/WMM/data/WMM2015/

[3] Yanovskii, B.M. (1978) Earth Magnetism. Publishing House of Leningrad University, Leningrad.

[4] Ivanchin, A. (2014) Equilibrium Elastic Stress Field of the Earth's Solid Shell. Internatiol Journal of Geoscience, 5, 464-473. https://doi.org/10.4236/ijg.2014.54044

[5] Ivanchin, A. (2015) Physics versus Geophysics. LAP LAMBERT, Saarbruecken.

[6] Ziman, J.M. (1995) The Theory of Solids. University Press, Cambridge.

[7] Kuznetsov, V.V. (1990) Physica of the Earth and the Solar System. Institute of Geology and Geophysics, Novosibirsk.

[8] Ivanchin, A. (2011) Nonpotential Solution of the Electron Problem. 
A. Ivanchin

https://arxiv.org/abs/0902.1286

[9] Ivanchin, A. (2017) Electrostatic Theory of Elementary Particles. World Journal of Nuclear Science and Technology, 7, 232-251.

https://doi.org/10.4236/wjnst.2017.74019 